\title{
La memoria como criterio de valoración de calidad en el ciberperiodismo: algunas consideraciones
}

\author{
Por Marcos Palacios
}

\begin{abstract}
Resumen: La consolidación de internet como soporte para la actividad periodística trajo consigo en los últimos años la necesidad de evaluar la calidad de los productos disponibles en la Web. La traslación a internet de criterios de evaluación, metodologías y herramientas empleadas en el análisis de calidad de los productos impresos, no tiene en cuenta las especificidades importantes de los productos online. Por otro lado, las herramientas que ya existen para la evaluación de sitios web son de carácter genérico, lo que conlleva resultados que no contemplan características propias del hacer periodístico. Este artículo explora algunas de esas dificultades y utiliza el ejemplo de la memoria extendida para indicar algunos de los desafios que se presentan para los analistas de productos periodísticos desarrollados para las redes telemáticas de alta velocidad.

Palabras clave: Periodismo, Periodismo en internet, Calidad, Memoria.

Title: Memory as a criterion for quality assessment in online newspapers: some considerations

Abstract: The consolidation of cyberjournalism as a new form of journalistic production in recent years brought about the need to evaluate the quality of newspapers and magazines produced for the Web. Using the same concepts and analytic tools developed for the traditional press in the evaluation of cyberjournalism leads to the undervaluation of specific characteristics of journalistic products designed for the new medium. On the other hand, analytic tools used for website evaluation are generally too generic and ill adapted for the specificities of cyberjournalism. In this article we explore some of the difficulties involved in quality assessment of online journalism. The difficulties in analyzing memory in cyberjournalism is used to indicate some of the challenges posed for the webnewspaper analyst.
\end{abstract}

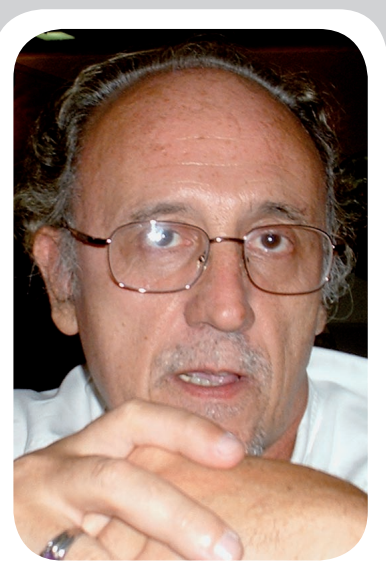

Marcos Palacios es profesor titular de periodismo en la Facultad de Comunicación de la Universidade Federal da Bahia (Brasil), investigador senior del CNPq (Conselho Nacional de Desenvolvimento Científico e Tecnológico ) y del Programa de Pós-Graduação em Comunicação e Cultura Contemporâneas da Universidade Federal da Bahia.

Keywords: Journalism, Cyberjournalism, Journalism and internet, Memory.

Palacios, Marcos. "La memoria como criterio de valoración de calidad en el ciberperiodismo: algunas consideraciones". El profesional de la información, 2009, mayo-junio, v. 18, n. 3, pp. 270-276.

DOI: 10.3145/epi.2009.may.03

Nuestro esfuerzo en este escrito se centra en señalar algunas de las dificultades que afronta la evaluación de la calidad de los productos ciberperiodísticos.

La memoria se puede definir en una aproximación inicial como la capacidad de recuperación de informaciones anteriormente producidas sobre el asunto y relevantes para contextualizar y/o mejor encuadrar la noticia. Es una característica que está presente en cierta medida en soportes periodísticos tradicionales (prensa gráfica, radio, TV) pero que se encuentra tremendamente potenciada en la Web, y será empleada como ejemplo para indicar algunas de las novedades e innumerables desafíos que representa para el analista del ciberperiodismo.

Si aceptamos la noción de que "calidad es una síntesis a partir de un conjunto de características percibidas por el espíritu"1, la memoria se presenta y se suma como elemento nuevo y necesario al cuadro de atributos a ser examinados en cualquier tentativa de establecer patrones y rankings de calidad para las publicaciones online.

"La memoria es la capacidad de recuperación de informaciones anteriormente producidas y relevantes para contextualizar y/o mejor encuadrar la noticia"

Es un elemento nuevo, porque está ausente de los conjuntos de atributos tradicionalmente considerados como parte en marcos de referencia para la valoración de calidad en productos periodísticos disponibles en otros soportes (prensa, radio, TV). 
Es un elemento reciente, por estar directamente asociado a la difusión y generalización del uso de las bases de datos como característica del ciberperiodismo en la fase de las conexiones rápidas (Machado, 2001, 2004; Barbosa, 2004, 2008a, 2008b; Fidalgo, 2004).

Es un elemento necesario, por presentarse como un factor de ruptura en el conjunto de particularidades específicas del periodismo producido en redes digitales, una vez que la memoria en el nuevo soporte se va a presentar como "múltiple, instantánea y acumulativa" (Palacios, 2004; Canavilhas, 2004; Mielniczuck; Barbosa, 2005; Silva-Junior, 2006) y, por tanto, extremadamente potenciada con relación a su expresión en soportes periodísticos anteriores.

Esa memoria potenciada es un ejemplo claro de una propiedad (de entre varias: hipertextualidad, multimedialidad, interactividad, personalización, actualización, etc.) que marca especificidades del ciberperiodismo e ilustra cómo la simple transposición y uso de instrumentos utilizados para análisis en otros soportes se muestran insuficientes cuando nos proponemos una tarea de evaluar y comparar productos periodísticos disponibles en redes telemáticas de alta velocidad.

Como enfatizábamos en nuestro trabajo de 2004, en el periodismo practicado en internet: "de la misma forma que la 'quiebra de los límites físicos' en la Web posibilita la utilización de un espacio prácticamente ilimitado para disposición de material noticioso, sobre los más variados formatos (multi)mediáticos, se abre la posibilidad de disposición online de toda la información anteriormente producida y almacenada, mediante la creación de archivos digitales con sistemas sofisticados de indexación y recuperación de la información" (Palacios, 2004) ${ }^{2}$.

Como consecuencia señalábamos que "en la Web, la conjugación de memoria con instantaneidad, hipertextualidad e interactividad, bien como la inexistencia de limitaciones de almacenamiento de información, potencian de tal forma la memoria que es legítimo afirmar que tenemos en esa combinación de características y circunstancias una ruptura con relación a los soportes mediáticos anteriores (Palacios, 2004) . $^{3}$

Melinda McAdams (1995), en su artículo sobre la construcción de la versión online del Washington Post, ya señalaba la importancia de los archivos como valor agregado y modelo de negocio (puntualmente sobre el cobro para consultas de artículos anteriores a 1986), llamando la atención sobre las entusiastas respuestas de los lectores en relación a la colocación online de los archivos y sus quejas sobre el cobro por el acceso.

A medida que avanzaron los recursos técnicos disponibles para la producción del periodismo en la Web y, sobre todo, en la medida que los archivos se volvían cada vez más operacionales en función de la conjugación de la Web con las bases de datos (Machado, 2001, 2004; Barbosa, 2004, 2008a, 2008b; Fidalgo, 2004), la memoria pasó a ser incorporada al hacer periodístico en la Web; bien como recurso de contextualización/ampliación del material noticioso diario, o en "especiales" y reportajes en profundidad (Ribas, 2008).

Un problema que está presente desde siempre con relación a la valoración de la calidad en productos periodísticos en la Web es el hecho de que los instrumentos disponibles, tanto teóricos como empíricos (cuestionarios, fichas de valoración, etc.) han sido, en su gran mayoría, creados y formateados para el análisis de sitios web en general, y no para el periodismo en particular ${ }^{4}$. Tal situación es claramente discernible, por ejemplo, cuando examinamos los modelos de análisis heurístico de Nielsen y sus propuestas de valoración (Nielsen, 1999, 2001, 2004).

En el panorama brasileño, un trabajo de Carrato (2001), orientado a la evaluación de la prensa de $M i$ nas Gerais, compara básicamente versiones online e impresas de periódicos "mineros", sin ninguna mención al uso de archivos como factor diferencial. David (2004) presenta una reflexión sobre el uso ampliado de la memoria en el periodismo online. Su aportación merece atención por los diversos puntos que recoge para una posible incorporación de la memoria como criterio diferencial en la valoración de calidad, sin embargo no propone instrumentos específicos para su medición. Galarça (2005), utilizando un referencial semiótico, refuerza la idea de la inexistencia de criterios consensuados para evaluar la calidad y resalta la multimedialidad como factor diferencial de calidad para las publicaciones periodísticas online. En el trabajo de Galarça, una vez más, la dimensión de la memoria está ausente.

"La memoria potenciada es una propiedad específica del ciberperiodismo, como la hipertextualidad, multimedialidad, interactividad, personalización o actualización."

La percepción de que eran necesarios modelos específicamente creados para el análisis de productos periodísticos en la Web, así como la inclusión de la memoria como indicador de calidad, ya estaba presente en un estudio -todavía incipiente- llevado a cabo por el GJOL (Grupo de Pesquisa em Jornalismo Online) de la Universidad Federal de Bahía, en 2002. En ese trabajo, de análisis y mapeo de las características entonces 
identificables en periódicos online brasileños y portugueses, la memoria (existencia de archivos) fue incluida entre los elementos investigados (Mielniczuk et al., 2002). La misma preocupación se va a evidenciar en el trabajo de Machado (et al., 2003) sobre patrones del periodismo online en Bahía. En un estudio realizado en 2004, Hope y Li -usando una clasificación de Zhang y Dran (2000) - propusieron una lista de factores identificables en un sitio web divididos en dos grupos: factores de higiene (Hygiene Factors) y factores motivadores (Motivador Factors). Los factores de higiene son los elementos cuya presencia en la web es funcional y de servicio y cuya ausencia causa insatisfacción; los factores de motivación son aquellos que agregan valor a una web y contribuyen a la satisfacción del usuario. La referencia a la memoria como factor motivador apareció sobre la rúbrica general archives (archivos) recibiendo puntuación relativamente baja en la percepción de los participantes del estudio en relación a su importancia en el conjunto de factores propuestos para la valoración de calidad.

\section{Experiencia reciente e indicaciones}

En el marco de una investigación comparativa entre los cibermedios brasileños y españoles (Convenio de colaboración Brasil-España para el estudio de los cibermedios, CAPES/DGU 140/07), fue preciso el establecimiento de una metodología que permitiese la comparación de los medios de ambos países. Ante la escasez de modelos aplicables y de la existencia de una utilización anterior por el grupo de investigadores españoles participantes del convenio, se optó como punto de partida del estudio por la adopción del modelo de Lluis Codina (2003) para la evaluación de productos online.

La elección del cuestionario de Codina fue realizada con plena conciencia previa de las limitaciones del modelo, y con vistas a su utilización crítica y sujeta a modificaciones y ampliaciones.

La limitación mayor se debe justamente al hecho de que este modelo, como tantos otros, está dirigido al análisis de sitios web en general y no específicamente para la evaluación cualitativa de sitios periodísticos.

Además, como señala Saad (2008), persiste sin solución el problema de la subjetividad inherente a este tipo de estudios. Codina simplemente coloca la subjetividad y la intrasubjetividad como datos del proceso que deben ser aceptados. Así, respondiendo a la pregunta de cómo combatir la subjetividad, expresa: "Lo primero que hay que señalar es que no se puede eliminar del todo, sin embargo, se pueden hacer algunas cosas para reducirla al máximo o para rebajarla a un nivel anecdótico. Un método es la experiencia y otro la intersubje- tividad. Empecemos por el segundo, diversos estudios de usabilidad [...] se resuelven con la realización del mismo estudio por tres personas distintas [...] El método de la experiencia nos indica que es aconsejable que los evaluadores tengan un buen conocimiento del tema [...] una tercera opción puede consistir, simplemente, en retirar aquellos indicadores que se consideren excesivamente subjetivos si el contexto del tipo de evaluación lo permite. (Codina, 2003: 166)

El modelo de cuestionario de Codina fue trabajado por el grupo de investigadores de Brasil y de España, con el objetivo de adaptarlo -sin eliminar sus líneas fundamentales- para la evaluación de las especificidades de los sitios periodísticos.

A partir del original, el cuestionario fue rediseñado tras un análisis crítico. La eficacia del nuevo modelo resultante fue comprobada mediante su aplicación a una publicación online (diario Público, de Portugal), el 15 de mayo de 2008. Tras el análisis de los resultados obtenidos de la prueba realizada sobre el periódico portugués, se produjo una segunda versión que se aplicó en siete periódicos brasileños y ocho periódicos españoles el 10 de septiembre de $2008^{5}$. La aplicación del cuestionario a los 15 medios se llevó a cabo en dos horarios previamente fijados (9:00 horas y 20:00 horas de los respectivos horarios locales de Brasil y de España), con el fin de incluir entre los propósitos del estudio las variaciones detectables entre las ediciones analizadas en los dos momentos diferentes.

Los primeros análisis y reflexiones que siguen a la aplicación del cuestionario de Codina modificado, tienden a indicar que puede ser útil para identificar, en líneas generales, "el estado de la web" de cada periódico relacionándolo con lo que antes denominamos formas "consensuadas" o "intersubjetivas" más genéricas de medición. O sea, es posible establecer la posición relativa de cada diario cuando se los compara, mediante indicadores de carácter más universal y genérico, aplicables a cualquier tipo de sitio web.

Se observa cómo la nueva herramienta es útil para el establecimiento de valoraciones preliminares, así como para la detección de aquellos problemas más evidentes, pero cuando se quiere analizar lo que podemos denominar especificidades de las web periodísticas, los límites del instrumento de Codina se hacen más visibles. Es decir, a pesar de detectar aspectos de la estructuración en páginas del sitio web y de la propia estructuración de contenidos, que son de interés central para el análisis de productos ciberperiodísticos en la medida en que permite una aproximación a las características asociadas a la escritura periodística en la Web (hipertextualidad, multimedialidad, interactividad, personalización, actualización, memoria $)^{6}$, esta metodología demuestra 
ser limitada al no hacer posible el análisis de las formas específicas que tales elementos asumen en el texto ciberperiodístico tomado en su dimensión histórica.

Para ejemplificar el problema estudiado, tomamos una de las propiedades de los cibermedios, la memoria, y examinamos cuál es su tratamiento analítico por medio de la herramienta/cuestionario propuesta por Codina (2003).

En el formulario original la memoria aparece sobre la rúbrica archivos, siendo analizada de manera binaria:

- Posibilidad de consulta a ejemplares anteriores (sí o no)

- Existencia de hemeroteca histórica (sí o no)

En caso positivo: acceso gratuito o acceso de pago.

En caso de acceso libre: necesidad o no de registro del usuario.

\section{"En nuestra propuesta se incluye el requisito: ¿ítems de archivo se incorporan como contenido relacionado a las noticias? (sí o no)"}

Como se observa, la concepción subyacente es de un archivo estático, que puede apenas ser consultado, no habiendo apertura para un análisis de una utilización dinámica e integrada de los recursos de memoria, es decir, no se contempla un análisis de los textos producidos y el uso de la memoria (bases de datos) en la construcción de la información periodística (narratividad). Posteriormente a la aplicación del instrumento piloto, en el ámbito del Convenio Brasil-España, fue propuesta una reformulación en el sentido de incorporar también una verificación -todavía sumaria- de existencia de memoria integrada/dinámica, con la inclusión de un requisito sobre el uso efectivo del archivo en los textos producidos:

¿Ítems de archivo se incorporan como contenido relacionado a las noticias disponibles en el vehículo analizado? (sí o no)

Se trata de un paso inicial, que hace posible un mejor encuadre de la memoria en cuanto criterio de determinación de calidad, pero que ciertamente todavía está lejos de dar cuenta de todos los aspectos relacionados con "formas específicas" de utilización de la memoria en el texto periodístico (Canavilhas, 2004; Palacios,
2004: David, 2004; Mielniczuck; Barbosa, 2005; Ribas, 2008), especialmente en lo que respecta a su función de elemento esencial de creación de contexto.

A título de ilustración de los avances de la incorporación de la memoria en publicaciones periodísticas online, indicamos tres ejemplos de usos recientes en tres cibermedios de importancia.

En la figura 1, tenemos un ejemplo relativamente simple de incorporación de memoria al texto periodístico en el Estadão.com.br (Brasil) mediante la recuperación de material de archivo (base de datos) del propio periódico en base a palabras claves agregadas a la noticia. Una nota sobre legislación acerca del aborto en Uruguay (19/11/2008) recuperó 96 textos relacionados, mediante la etiqueta aborto, colocada al final de la noticia original.

En la figura 2 tenemos un ejemplo más sofisticado, extraído de la edición online del New York Times (EUA) (19/11/2008) en el cual una noticia sobre la pérdida de influencia político-electoral del sur norteamericano

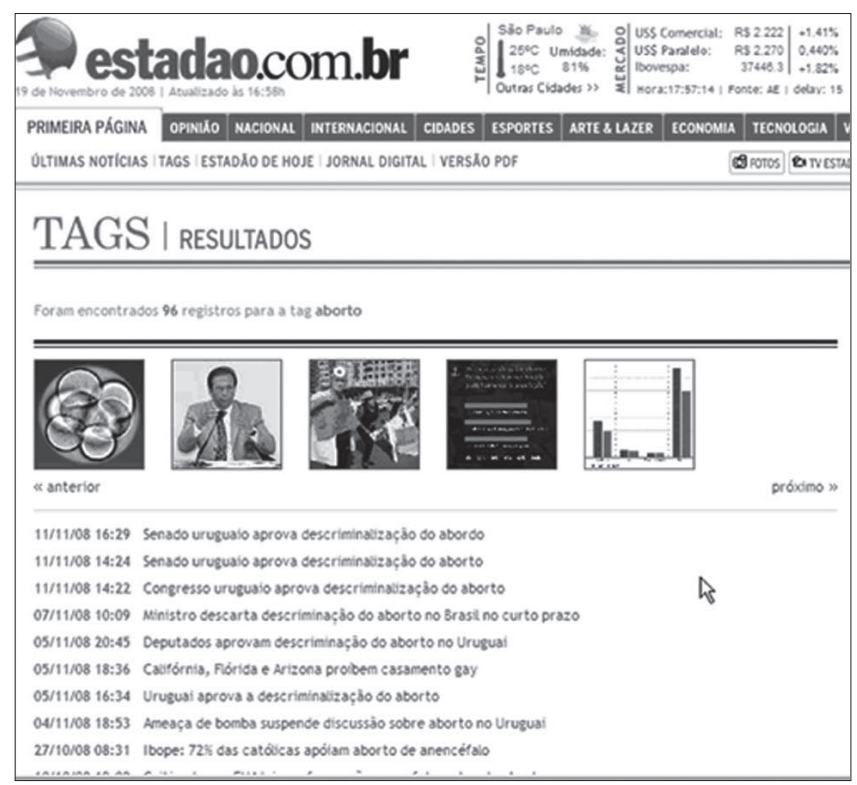

Figura 1. Recuperación de la memoria por medio de etiquetas

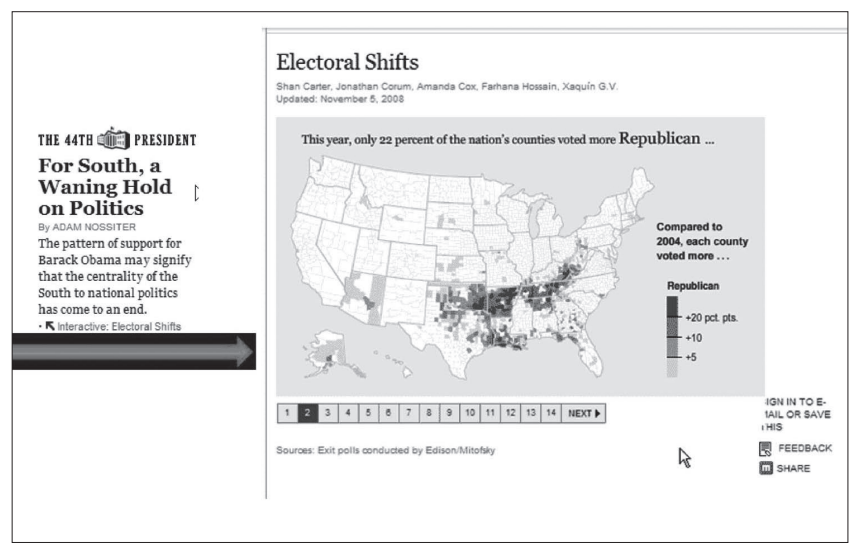

Figura 2. Recuperación gráfica de memoria 
permite acceder a una recuperación gráfica de memoria, mediante un registro histórico de la participación del sur en elecciones anteriores.

En la figura 3, un ejemplo extraído de El País (España) (19/11/2008) muestra una amplia complementación/contextualización por la adición de memoria obtenida gracias al recurso $A$ Fondo, que asocia noticias corrientes a material de archivo del periódico.

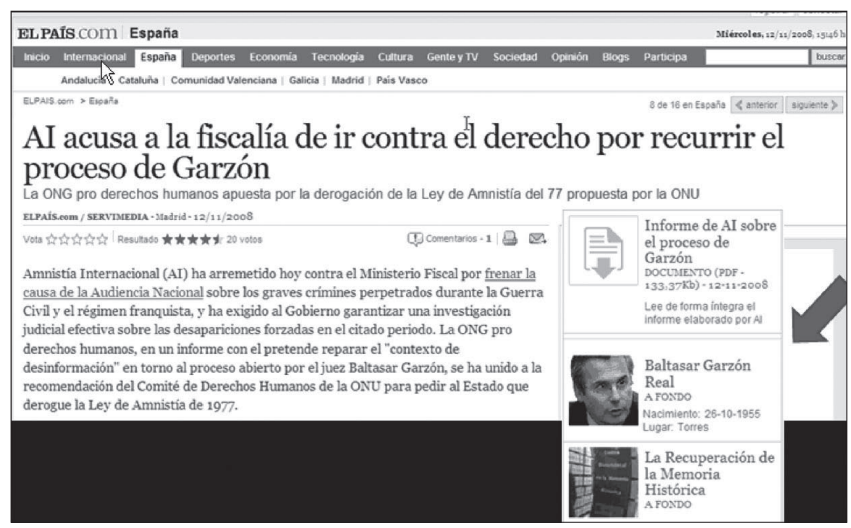

Figura 3. El recurso A Fondo, de El país, produce recuperación automática de memoria

Desafíos de naturaleza semejante pueden ser observados con respecto a la medición de una efectiva utilización de las demás particularidades usualmente asociadas al texto periodístico en la Web (hipertextualidad, multimedialidad, interactividad, personalización, actualización). Para cada uno de dichos ítems se presenta la tarea inevitable y el desafío de establecer indicadores específicos más amplios y detallados, que puedan traducir de forma más apropiada los niveles de su incorporación y efectiva utilización en la producción de un texto periodístico. Las dificultades de esta tarea son detectables hasta por el mismo carácter inconsistente, impreciso - tenemos plena conciencia de eso- de algunos de los términos que estamos utilizando en este artículo, como "amplios" o "apropiados", que obviamente requieren ser más explícitos y elaborados. Y tal elaboración solamente se puede lograr mediante la construcción experimental de nuevos instrumentos, en sucesivas aproximaciones y refinamientos conceptuales y pragmáticos.

Si nos mantuviéramos en los límites de nuestra definición general y mínima de calidad, presentada en el inicio de este artículo ("la calidad es el conjunto de propiedades y características de un producto o servicio que le confiere su aptitud para satisfacer necesidades explícitas o implícitas") parece inevitable que el avance de la "alfabetización digital" de los usuarios de la Web reclame (explícita o implícitamente) un grado de sofisticación cada vez mayor del producto periodístico ofrecido en lo que respecta a las formas específicas de utilización de las posibilidades y potencialidades propuestas por la nueva plataforma de producción.

\section{Conclusiones y recurrencias}

Intitulado como consideraciones, este breve artículo no puede tener pretensiones de conclusión en el sentido estricto del término. Señalaremos tan sólo algunos elementos recurrentes que deben figurar en reflexiones futuras sobre la inclusión del ítem memoria en la valoración de calidad de los periódicos producidos en redes telemáticas.

Las crecientes posibilidades abiertas para la recuperación de memoria, a partir de las sofisticadas bases de datos en la producción periodística, tiene efectos que pueden y deben ser considerados:

- en las rutinas productivas en las redacciones, con la creciente facilidad de consultas y apropiación de informaciones en bases de datos internas y externas al vehículo,

- en los modelos de negocios, con una vasta gama de posibles incorporaciones de elementos de memoria como parte del negocio establecido para los periódicos (acceso pago total o parcial, conjugación de bases de datos/memoria/publicidad, otras formas de agregación de valor),

- en la producción de formatos narrativos diferenciados, con distintas formas de incorporación de memoria (background, contexto, contraposición, etc.),

- en las formas de interacción con el usuario, que pasa a disponer de recursos para investigar, en la propia web del periódico, aspectos históricos o de contextualización alrededor del material de actualidad que se le ofrece, ya sea eventualmente personalizando su memoria en espacios de la propia web periodística que utiliza.

\section{"Es necesario ampliar el concepto de memoria más allá de la dimensión del archivo (existencia o no, acceso gratuito o pago, etc.)"}

De las afirmaciones anteriores resulta, como apuntes para futuras investigaciones sobre calidad en los productos periodísticos:

- la necesidad de superar la escasa atención que el ítem viene recibiendo en los estudios hasta aquí realizados, tanto en el entorno nacional como internacional, 
- la necesidad de ampliar el concepto de memoria, una vez que en los casos en que haya algún tipo de inclusión de ese ítem en la lista de criterios utilizados su apreciación queda restringida a la dimensión del archivo (existencia o no, acceso gratuito o pago, extensión temporal del material dispuesto, etc.),

- la necesidad de considerar las posibilidades de personalización de memoria -por medio de la creación de áreas de lectores-, donde la recopilación de noticias y enlaces personalizados constituyan nuevas formas de utilización de la memoria, como adición de valor al material periodístico originalmente producido.

A pesar de la evidente dificultad de apropiación socio-técnica de la memoria en los cuestionarios de observación de contenidos empleados en la determinación de corpora de larga escala (como es el caso del modelo propuesto por Codina), es preciso buscar la máxima viabilidad de valoraciones de ese ítem ya en la etapa de mapeo -representado por la aplicación de cuestionarios-, para que en una etapa siguiente esos refinamientos puedan ser realizados mediante abordajes más cualitativos, in loco y para corpora más reducidas.

\section{Notas}

1. Conforme el Diccionario Michaelis de la Lengua Portuguesa. La Norma ISO 8402 establece que: "La calidad es el conjunto de propiedades y características de un producto o servicio que le concede su aptitud para satisfacer necesidades expresas o implícitas" (Jiménez-Piano; Ortiz-Repiso, 2007: 53).

2. "Da mesma forma que a "quebra dos limites físicos' na Web possibilita a utilização de um espaço praticamente ilimitado para disponibilização de material noticioso, sob os mais variados formatos (multi)mediáticos, abre-se a possibilidade de disponibilização online de toda informação anteriormente produzida e armazenada, através da criação de arquivos digitais, com sistemas sofisticados de indexação e recuperação da informação (Palacios, 2004).

3. (...) que na Web, a conjugação de Memória com Instantaneidade, Hipertextualidade e Interatividade, bem como a inexistência de limitações de armazenamento de informação, potencializam de tal forma a Memória que é legítimo afirmar-se que temos nessa combinação de características e circunstâncias uma Ruptura com relação aos suportes mediáticos anteriores (Palacios, 2004).

4. Véase el sumario de referencias sobre análisis de sitios web en general en Hope y Li (2004).

5. Los periódicos incluidos en el estudio fueron los siguientes: Por el lado brasileño: Folha de São Paulo (San Pablo), Diário de Santa Catarina (Florianópolis),
Gazeta do Povo (Curitiba), Jornal do comércio (Recife), Zero hora (Porto Alegre), A tarde (Salvador) y $O$ correio Brasiliense (Brasilia). Por la parte española tenemos: Sur (Andalucía), El correo (Bilbao), Diario de Navarra, La Vanguardia, La voz de Galicia, Las provincias o Levante (Valencia), El mundo y El país.

6. Para una aproximación inicial a los estudios que inciden sobre aspectos de caracterización del texto periodístico en la Web y sus fases o etapas de desarrollo, a partir de la óptica de una vasta gama de autores, recomendamos como un posible punto de partida la ya considerable producción de los integrantes del GJOL (Grupo de Pesquisa en Jornalismo Online). http://www.facom.ufba.br/jol

\section{Bibliografía}

Barbosa, Suzana. "Jornalismo digital em ambientes dinâmicos. Propriedades, rupturas e potencialidades do Modelo JDBD". Observatorio (OBS*) Journal, 2008a, n. 4, pp. 217-244.

http://obs.obercom.pt

Barbosa, Suzana. "Modelo JDBD e o ciberjornalismo de quarta geração". En III Congreso Intl. de Periodismo en la Red - Foro Web 2.0: Blogs, wikis, redes sociales y e-participación, GT 7 - Cibercultura y tendencias de la prensa en internet. Facultad de Ciencias de la Información, Universidad Complutense de Madrid, $2008 \mathrm{~b}$.

Barbosa, Suzana. "Identificando remediações e rupturas no uso de bancos de dados no jornalismo digital". En Anais do II SBPJor. Salvador-BA/Brasil, 2004.

Bogart, L. Press and public: who reads what, when, where, and why in American newspapers ( $2^{\text {nd }}$ ed.). Hillsdale, NJ: Lawrence Erlbaum and Associates, 1989.

Bogart, L. "Reflections on Content quality in newspapers". Newspaper research journal, Winter, 2004.

http://findarticles.com/p/articles/mi_qa3677/is_200401/ai_n9353031

Canavilhas, João. A internet como memória. UBI/Bocc, 2004.

http://www.bocc.ubi.pt/pag/canavilhas-joao-internet-como-memoria.pdf

Carrato, Ángela. Jornal online, uma avaliação sobre a experiência mineira. Intercom, 2001.

Codina, Lluís. "Hiperdocumentos: composición, estructura y evaluación". En: Díaz-Noci, Javier; Salaverría, Ramón (eds.) Manual de redacción ciberperiodística. Barcelona: Ariel, 2003.

David, Mónica. "Conexões de Memória: os testemunhos, os dados e o jornalismo online". En Congresso Ibero-Americano de Periodismo em Internet, Salvador, 2004.

http://www.espacioblog.com/myfiles/ciberperiodismo/AIAPI\%202004\%20 Monica\%20David.pdf

Fidalgo, António. "Do poliedro à esfera: os campos de classificação. A resolução semântica no jornalismo online”. En Anais do II SBPJor. SalvadorBA/Brasil, 2004.

Galarça, Sandro. Jornalismo online: como os internautas catarinenses avaliam duas propostas diferentes na internet. Anais. São Paulo: Intercom, 2005. www.walterlima.jor.br/academico/alcar/textos/midia_digital_sandro.doc

Georgia Scholastic Press Association. Newspaper evaluation guidebook, Henry W. Grady School of Journalism http://www.grady.uga.edu/gspa/Forms/WEBNewspaper.pdf

Gladney, G. A. "Newspaper excellence: How editors of small and large papers judge quality”. Newspaper research journal, 1990, v. 11, n. 2, pp. 58-71.

Hope, B.; Li, Z. "Online newspapers: the impact of culture, sex, and age on the perceived importance of specified quality factors". Information research, 2004, v. 9, n. 4.

http://informationr.net/ir/9-4/paper197.html\#Zhang\#Zhang 
Jiménez-Piano, Marina; Ortiz-Repiso, Virgínia. Evaluación y calidad de sedes web. Gijón: Ediciones Trea, 2007.

Machado, Elías. O jornal digital como epicentro das redes de circulação de notícias, 2001.

Machado, Elías. "Banco de dados como formato no jornalismo digital”. En Anais VI Lusocom, (CD-ROM), Covilhã, Portugal, abril, 2004a.

Machado, Elías; Borges, Clarissa; Miranda, Milena. "Modelos de produção de conteúdo no jornalismo digital baiano". Em: Machado, Elias; Palacios, Marcos (eds.). Modelos de jornalismo digital. Salvador: Calandra, 2003.

McAdams, Melinda. "Inventing an online newspaper", Interpersonal computing and technology: an electronic journal for the 21st Century, July, 1995, v. 3, n. 3, pp. 64-90.

http://www.helsinki.fi/science/optek/1995/n3/mcadams.txt

Meyer, Philip; KIM, Koang-Hyub. Quantifying newspaper quality: “I know it when I see it", paper delivered to the Newspaper Division, Association for Education in Journalism and Mass Communication, Kansas City, Mo., July 3, 2003.

Mielniczuk, L.; Barbosa, S. "Digital journalism. Democratizing social memory. Brazilian journalism research, v. 1, n. 2, semester 2, 2005.

http://www.unb.br/ojsdpp/viewissue.php?id=2

Mielniczuk, L.; Barbosa, S.; Ribas, B.; Narita, S.; Palacios, M. "Um mapeamento de características e tendências no jornalismo online brasileiro e português". Comunicarte, revista de comunicação e arte, v. 1, n. 2, Universidade de Aveiro, Portugal, set. 2002.

Nielsen, J. "Designing Web usability: the practice of simplicity", New Riders, 1999.

Nielsen, J. Ten usability heuristics.

http://www.useit.com/papers/heuristic/heuristic_list.html

Nielsen, J.; Tahir, M. "Homepage usability: 55 Websites deconstructed". New Riders, 2001.

Palacios, M. "Ruptura, continuidade e potencialização no jornalismo on- line: o Lugar da memória". En Machado, Elias; Palacios, Marcos (eds.), Modelos do jornalismo digital. Salvador: Calandra, 2004

http://www.facom.ufba.br/jol/pdf/2003_palacios_olugardamemoria.pdf

Palacios, M. et al. "Um jornal laboratório multimídia, multi-usuário e descentralizado. O caso da Plataforma Panopticon". Pauta Geral, 2005, Ano 12, No 7. Salvador: Calandra. pp: 105-115,

Ribas, B. Memória e ciberjornalismo: considerações sobre potencialidades para a reconfiguração da prática jornalística nas redes digitais, texto submetido como requisito parcial para a Qualificaão Doutoral, Programa de Pós-Graduação em Comunicação e Cultura Contemporâneas, FACOM/ UFBA, Salvador, 2008.

Saad, Elizabeth Corrêa. Relatório integrador do teste-piloto da ficha de avaliação de qualidade dos cibermeios. Equipe brasileira do Convênio Capes-DGU, Documento Interno do Convênio CAPES/DGU 140/07, 2008.

Silva-Júnior, Afonso da. Uma trajetória em redes: modelos e características operacionais das agências de notícias, das origens às redes digitais, com três estudos de caso. (Tese de doutorado). FACOM/UFBA, Salvador. 2006

Zhang, P.; Dran, G. M. Satisfiers and dissatisfiers: a two-factor model for website design and evaluation. Journal of the American Society for Information Science, 2000, v. 51, n. 14, pp. 1253-1268

Ziller, Joana. "A qualidade de informação em webjornais - conceitos norteadores”. XXVIII Congresso Brasileiro de Ciências da Comunicação - Uerj -5 a 9 de setembro de 2005 ,

http://reposcom.portcom.intercom.org.br/dspace/bitstream/1904/16804/1/ R1699-1.pdf

\section{Traducción de Gonzalo Prudkin}

Marcos Palacios, Facultad de Comunicación, Universidad Federal de Bahia.

palacios@ufba.br

\section{Máster en Documentación Digital} Máster y Programas de posgrado on line

Arquitectura de la información en sitios web • Organización de la información y sistemas de gestión de base de datos $\bullet$ Buscadores y posicionamiento web $\bullet$ Documentación audiovisual $\bullet$ Web 2.0 (nuevo) $\bullet$ Archivística digital $\bullet$ Gestión de contenidos y gestión del conocimiento $\bullet$ Repositorios y bibliotecas digitales (ampliado) • Usabilidad.

Dirección: Cristòfol Rovira y Lluís Codina

Calendario: del 15 de octubre de 2009 al 30 de junio de 2010.

www.documentaciondigital.org

\section{Máster en Buscadores Marketing y Posicionamiento Máster y Programas de posgrado on line}

Fundamentos $\bullet$ Posicionamiento en Buscadores $\bullet$ Marketing en Buscadores $\bullet$ Vigilancia Tecnológica • Recuperación de la Información • Buscadores especializados.

Dirección: Cristòfol Rovira y Mari Carmen Marcos

Calendario: del 12 de noviembre de 2009 al 30 de junio de 2010.

www.masterenbuscadores.com

\section{Colaboran:}

Observatorio de Comunicación Científica $\cdot$ UPF

Instituto Universitario de Lingüística Aplicada · UPF

DOC 6 Consultores de Recursos de Información

Infórmate:

www.idec.upf.edu

Tel.: +34935421850

Balmes, 132-134

08008 Barcelona 


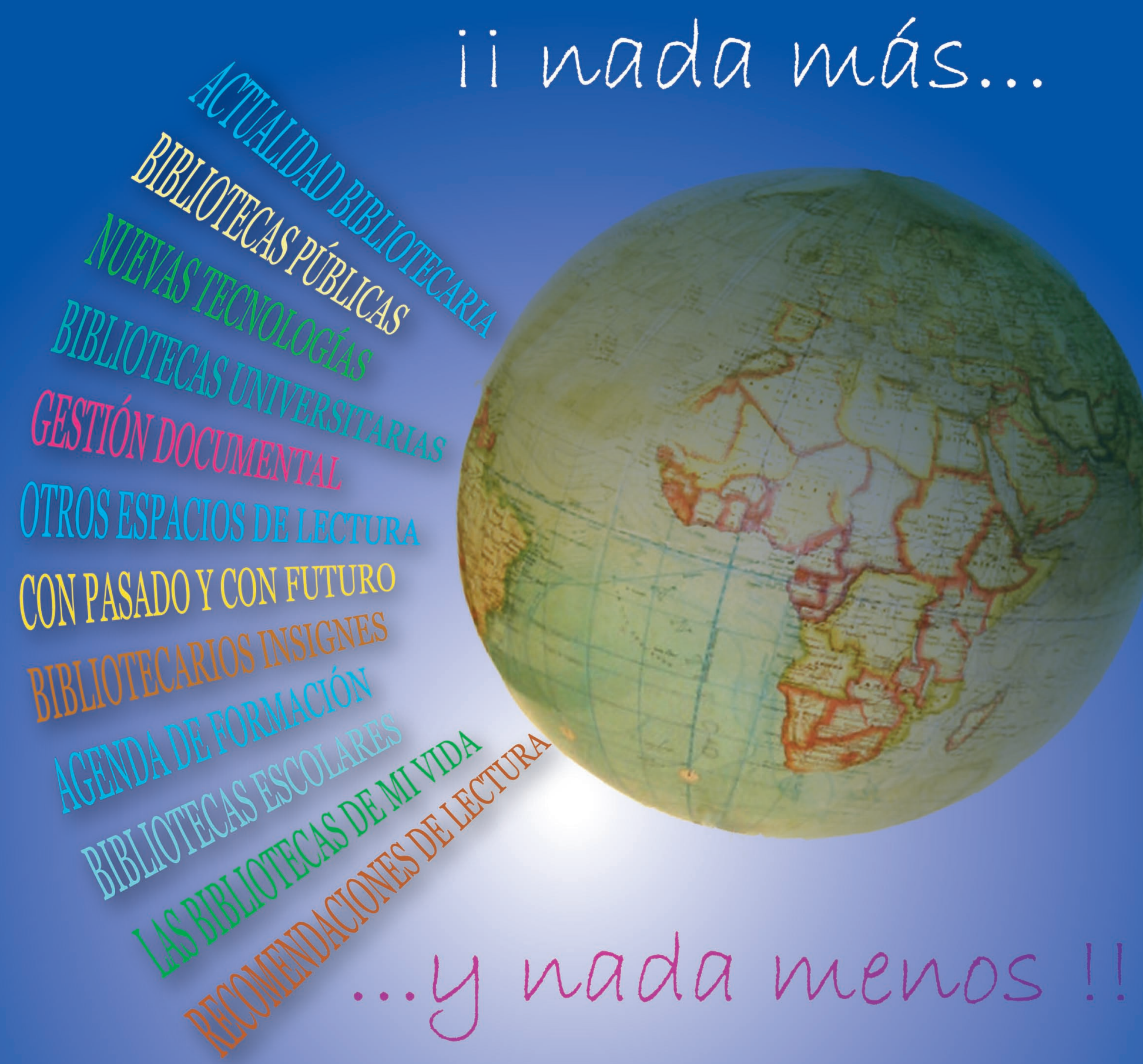

\section{...y nada menos!}

\section{Mi}

\section{La revista del mundo bibliotecario}

Suscríbete a Mi Biblioteca y recibirás cada año, de manera gratuita, el Calendario de la Lectura y el Anuario de Bibliotecas Españolas de la Fundación Alonso Quijano.

Tfno. 952235405 www.mibiblioteca.org 